\title{
Dysregulation of post-transcriptional modification by copy number variable microRNAs in schizophrenia with enhanced glycation stress
}

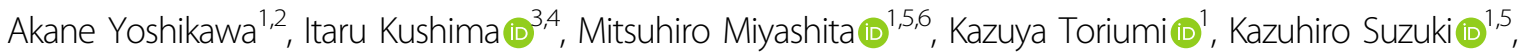 \\ Yasue Horiuchi (1) ${ }^{1}$, Hideya Kawaji $\mathbb{1}^{7}$, Shunya Takizawa ${ }^{8}$, Norio Ozaki ${ }^{3}$, Masanari Itokawa ${ }^{1}$ and Makoto Arai (1)
}

\begin{abstract}
Previously, we identified a subpopulation of schizophrenia (SCZ) showing increased levels of plasma pentosidine, a marker of glycation and oxidative stress. However, its causative genetic factors remain largely unknown. Recently, it has been suggested that dysregulated posttranslational modification by copy number variable microRNAs (CNV-miRNAs) may contribute to the etiology of SCZ. Here, an integrative genome-wide CNV-miRNA analysis was performed to investigate the etiology of SCZ with accumulated plasma pentosidine (PEN-SCZ). The number of CNV-miRNAs and the gene ontology $(\mathrm{GO})$ in the context of miRNAs within CNVs were compared between PEN-SCZ and non-PEN-SCZ groups. Gene set enrichment analysis of miRNA target genes was further performed to evaluate the pathways affected in PEN-SCZ. We show that miRNAs were significantly enriched within CNVs in the PEN-SCZ versus non-PEN-SCZ groups ( $p=0.032$ ). Of note, as per $\mathrm{GO}$ analysis, the dysregulated neurodevelopmental events in the two groups may have different origins. Additionally, gene set enrichment analysis of miRNA target genes revealed that miRNAs involved in glycation/oxidative stress and synaptic neurotransmission, especially glutamate/GABA receptor signaling, were possibly affected in PEN-SCZ. To the best of our knowledge, this is the first genome-wide CNV-miRNA study suggesting the role of CNV-miRNAs in the etiology of PEN-SCZ, through effects on genes related to glycation/oxidative stress and synaptic function. Our findings provide supportive evidence that glycation/oxidative stress possibly caused by genetic defects related to the posttranscriptional modification may lead to synaptic dysfunction. Therefore, targeting miRNAs may be one of the promising approaches for the treatment of PEN-SCZ.
\end{abstract}

\section{Introduction}

Schizophrenia (SCZ) is a neurodevelopmental disorder with a worldwide prevalence of around $1.0 \%$, leading to abnormalities in function and connectivity of key brain regions ${ }^{1}$. The most common symptoms are auditory hallucinations, delusions, withdrawal, flat affect, lack of motivation, and disorganized thinking. The onset, during early adolescence, strongly impacts school life and

\footnotetext{
Correspondence: Makoto Arai (arai-mk@igakuken.or.jp)

'Schizophrenia Research Project, Department of Psychiatry and Behavioral

Sciences, Tokyo Metropolitan Institute of Medical Science, 2-1-6, Kamikitazawa, Setagaya-ku 156-8506 Tokyo, Japan

${ }^{2}$ Department of Adolescent Psychiatry, Nihonbashi Sun Clinic, 2-1-21,

Nihonbashi, Chuou-ku, Tokyo 103-0027, Japan
}

Full list of author information is available at the end of the article educational achievement, leading to lifelong social and/or occupational dysfunction. Accumulating evidence suggests that both genetic and epigenetic factors are involved in the etiology of $\mathrm{SCZ}^{2-7}$. An integrative genetic and epigenetic analysis would accelerate the elucidation of the etiology.

Recent advances in genomic technologies have facilitated large-scale genomic studies. Previous research has successfully uncovered the polygenic nature of multiple common risk variants ${ }^{4,5,8}$ as well as the involvement of rare but highly penetrant variants ${ }^{9,10}$, and structural genetic variants called copy number variations (CNVs) in $\mathrm{SCZ}^{11-15}$. In the largest-scale CNV study to date, using 41,321 subjects, deletions in 1q21.1, 2p16.3, 3q29, 
and 22q11.21 were confirmed as risk factors for SCZ with high odds ratios (ORs) ranging from 3.8-67. $7^{14}$. The Psychiatric Genomics Consortium (PGC)-CNV analysis group has demonstrated that genes disrupted by CNVs in SCZ are enriched within the synaptic networks ${ }^{14}$. Kushima et al. performed the largest genomewide CNV analysis in the Japanese population so far and confirmed an increased burden of rare exonic CNVs in 2458 SCZ and 1108 autism spectrum disorder (ASD) cases $^{16}$.

MicroRNAs (miRNAs) are a family of short ( 22 nt), single-stranded, non-coding RNAs primarily involved in the posttranscriptional downregulation of gene expression via binding to the $3^{\prime} \mathrm{UTR}$ of target genes ${ }^{17}$. CNVmiRNA genes generate the complexity of miRNA regulation and function ${ }^{17,18}$. Genomic CNVs can induce the aberrant expression of integral miRNAs and their target genes $^{18}$, resulting in the dysregulation of posttranslational modification, crucial for the adaptation to environmental factors. Recently, it has been shown that the genetically dysregulated posttranslational modification caused by CNV-miRNAs contribute to the etiology of neurodevelopmental disorders including SCZ, ASD, and intellectual disability (ID) $)^{19}$. For example, in the context of SCZ, miRNA contents within CNV regions were significantly enriched compared to those in control subjects $^{19}$. Additionally, in ASD, miRNAs present in CNV loci were shown to regulate synaptic transmission and may, thus, contribute to the disorder phenotype $e^{20}$.

Genetic heterogeneity is a hallmark of SCZ; the genetic architecture of this common disorder is largely com$\operatorname{plex}^{21,22}$. To overcome the heterogeneity, the identification of SCZ subpopulations presenting with disturbances of specific metabolic pathways is a promising strategy. We previously reported an SCZ subtype with enhanced glycation/oxidative stress that showed accumulated plasma pentosidine (PEN-SCZ) ${ }^{23}$. Although around $20 \%$ of SCZ patients were reported to be PEN-SCZ ${ }^{24}$, the genetic and epigenetic factors that account for the accumulation of pentosidine and their contribution to this phenotype have not yet been fully elucidated.

Here, we performed an integrative, genome-wide, CNV-miRNA analysis to investigate the genetic factors associated with SCZ with enhanced glycation/oxidative stress and to provide novel insights into the etiology of PEN-SCZ, focusing on the disturbance of posttranscriptional modification caused by CNV-miRNAs.

\section{Materials and methods Subjects}

In this study, 209 unrelated patients with SCZ were recruited, mainly from the Departments of Neuropsychiatry, Tokyo Metropolitan Matsuzawa Hospital, Takatsuki Hospital, Takatsuki Clinic, and RIKEN Brain
Science Institute near Tokyo, for both genetic and biochemical analyses. All subjects were ethnically Japanese. The patients were diagnosed according to the DSM-5 criteria (American Psychiatric Association) for SCZ or schizoaffective disorders as per the consensus of at least two experienced psychiatrists. Patients with a history of drug addiction or alcohol abuse/dependence were excluded. Patients with accompanying diabetes mellitus and chronic renal disease were also excluded because these diseases may affect the plasma pentosidine levels. Medical records were obtained with the approval of patients. The study was performed in accordance with the Declaration of Helsinki. This study was approved by the research ethics committee of each participating institute and written informed consent was obtained from all subjects. The subjects' demographics are presented in Table 1. Of note, to define the PEN-SCZ and non-PEN-SCZ cases, plasma concentration of pentosidine was measured via high-performance liquid chromatography as described previously ${ }^{25}$.

\section{Identification of CNV-miRNAs}

Genomic DNA was extracted from blood samples. Array comparative genomic hybridization (array CGH) was performed to identify rare $(<1 \%)$ CNVs in PEN-SCZ and non-PEN-SCZ patients; NimbleGen $720 \mathrm{~K}$ WholeGenome Tiling Arrays (Roche NimbleGen, Madison, WI, USA) were used for the CNV analysis. CNV calls were conducted using the Nexus Copy Number software v9.0 (Bio Discovery, El Segundo, CA, USA) with the Fast Adaptive States Segmentation Technique 2 algorithm. Quality control (QC) was performed as described previously $^{16,26}$. Briefly, QC scores for each sample based on the statistical variance of the probe-to-probe log ratios were calculated and samples with $\mathrm{QC}>0.15$ were removed. As sample QC, subjects with excessive numbers of autosomal $\mathrm{CNVs}$ were also removed from the analysis. After filtering out common CNVs $(\geq 1 \%)$, rare CNVs $(<1 \%)$ in 185 patients were used for further analyses. All genomic locations are given in GRCh38 coordinates, and gene annotation was based on GENCODE version 27. Of note, previously, we confirmed that $\mathrm{CNV}$ calls from NimbleGen arrays are highly accurate with a validation rate $>99 \%{ }^{27}$. The miRNAs present in CNVs were then identified via the analysis of the chromosomal coordinates as per the UCSC Genome Browser (http://genome.ucsc.edu/) using the sno/ miRNA prediction tracks of the Mar.2006 (NCBI36/ hg18) assembly.

\section{Prediction of miRNA target genes}

To understand the function of CNV-miRNAs, we assessed validated and putative miRNA target genes in the context of two miRNA databases, mirDB (http://www. 
mirdb.org/) ${ }^{28}$ and miRWalk 3.0 (http://mirwalk.umm.uniheidelberg.de $/)^{29}$. The validated gene targets of miRNAs were obtained using the filtering function in the miRWalk 3.0 database. GeneCards (https://www.genecards.org/) and STRING v11 (https://string-db.org//) 30 were used for the functional annotation of miRNA target genes. Additionally, the GTEx portal (https://gtexportal.org/home/) was used to examine the tissue-specific expression of the validated and/or putative miRNA target genes.

\section{Comparison of CNV-miRNAs in PEN-SCZ versus non-PEN- SCZ}

First, the average length of total CNVs in PEN-SCZ versus non-PEN-SCZ groups was compared. Second, the average number of genes within CNVs was obtained. Third, the total number of miRNAs within CNVs was compared between the two groups. Forth, the number of patients harboring CNV-miRNAs was determined. Fifth, the average number of miRNAs per patient was compared. Finally, the number of patients with rates of miRNA genes per total genes within CNVs greater than $50 \%$ was compared between PEN-SCZ and non-PEN-SCZ groups.

\section{Gene ontology and pathway analyses}

Gene ontology (GO) analysis was performed with all miRNAs observed within CNVs in each group, using MetaCore (Clarivate Analytics), a web-based licensed tool for comprehensive pathway analyses ${ }^{31}$. Gene set enrichment analysis of miRNA target genes was further performed to characterize the pathways affected in the PENSCZ group, also using MetaCore ${ }^{31}$.

\section{Statistical analysis}

With respect to the demographic data, the average age, age of onset, plasma pentosidine levels, and the average of chlorpromazine equivalent doses were compared using the Student's $t$-test (two-tailed). The rate of treatment resistant SCZ between PEN-SCZ and non-PEN-SCZ was evaluated by one-sided Fisher's exact test. Regarding the CNV-miRNA analysis, the average length of total CNVs, number of genes within CNVs, and number of miRNAs per patient were assessed by Student's $t$-test. To evaluate the differences in the rate of CNV-miRNAs between PENSCZ and non-PEN-SCZ groups, one-sided Fisher's exact tests were used. If no variants were found in the two-bytwo table, the OR was calculated after a 0 cell correction (0.5 was added to all cells), conducted to reduce bias ${ }^{26}$.

Table 1 Demographics and summary of miRNAs within CNVs in schizophrenia patients with/without accumulated plasma pentosidine.

\begin{tabular}{|c|c|c|c|}
\hline \multirow{2}{*}{$\begin{array}{l}\text { Group } \\
\text { Pentosidine }\end{array}$} & \multirow{2}{*}{$\begin{array}{l}\text { PEN-SCZ } \\
\text { High }\end{array}$} & \multirow{2}{*}{$\begin{array}{l}\text { non PEN-SCZ } \\
\text { Normal }\end{array}$} & \multirow[t]{2}{*}{$P$ value/FC } \\
\hline & & & \\
\hline Number of patients & 94 & 91 & \\
\hline Age $\left(\right.$ mean \pm S.D. $\left.{ }^{\circ}\right)$ & $52.0 \pm 11.2$ & $47.0 \pm 14.1$ & 0.0026 \\
\hline Sex (Male/Female) & $50 / 44$ & $46 / 45$ & 0.68 \\
\hline Ethnicity & Japanese & Japanese & \\
\hline Age of onset (mean \pm S.D. ${ }^{\complement}$ ) & $25.7 \pm 9.4$ & $25.24 \pm 8.4$ & 0.37 \\
\hline Plasma pentosidine level (mean \pm S.D. $\left.{ }^{c}\right)\left(\mathrm{ng} / \mathrm{ml}^{\mathrm{d}}\right)$ & $128.1 \pm 126.0$ & $39.8 \pm 9.3$ & $5.19 \times 10^{-10}$ \\
\hline Average length of total CNV (Mb) & $3.6 \pm 21.9$ & $0.4 \pm 0.5$ & $0.083 / 8.4^{\mathrm{e}}$ \\
\hline Average of chlorpromazine equivalent doses & $1147.58 \pm 864.02$ & $716.43 \pm 607.65$ & $7.33 \times 10^{-5}$ \\
\hline The rate of treatment resistant SCZ (\%) & 39.3 & 11.9 & 0.0022 \\
\hline Average of number of genes within CNVs & $92.2 \pm 254.2$ & $4.5 \pm 7.6$ & $0.08 / 20.5^{e}$ \\
\hline Total number of miRNAs within CNVs & 205 & 21 & $9.8^{\mathrm{e}}$ \\
\hline Number of patients harboring CNV-miRNAs & $13(13.8 \%)$ & $13(14.3 \%)$ & 0.55 \\
\hline Average of the number of miRNAs per patient & 15.8 & 1.6 & $0.086 / 9.9^{e}$ \\
\hline Number of patients with the rate of miRNA genes per total genes within CNVs $\geq 50 \%$ & 5 & 0 & 0.032 \\
\hline Number of patients with the rate of miRNA genes per total genes within CNVs $<50 \%$ & 89 & 91 & \\
\hline
\end{tabular}

${ }^{a}$ Schizophrenia with accumulated plasma pentosidine level.

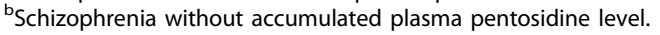

'Standard deviation.

${ }^{d}$ Cut off value; 55.2.

${ }^{e}$ Fold change. 


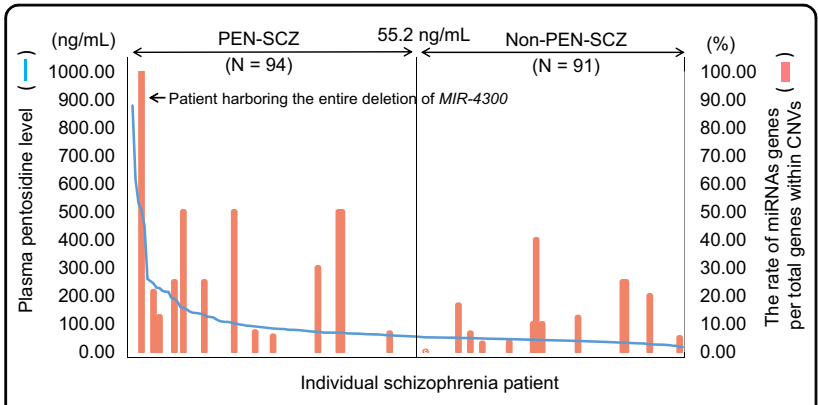

Fig. 1 Plasma pentosidine levels and the rate of miRNA genes per total genes within CNVs in schizophrenia patients. In the genome-wide CNV-miRNA analysis, we compared the rate of miRNA genes per total genes within CNVs in PEN-SCZ versus non-PEN-SCZ. MiRNA-enriched CNVs, defined as a rate of miRNA genes in genes within CNVs $>50 \%$, was significantly higher in PEN-SCZ versus nonPEN-SCZ $(p=0.032)$.

Additionally, with respect to the in silico prediction of miRNA target genes using miRWalk, the $p$ values were calculated from a random-forest-based approach using TarPmiR for miRNA target site prediction. A $p$ value of $<0.05$ was considered significant.

\section{Power analysis}

Power analysis was conducted using the Genetic Association Study (GAS) Power Calculator ${ }^{27,32}$. The following parameter assumptions were used to estimate power: ${ }^{27}$ prevalence of disease $=0.007$ and $\alpha=0.05$ in the context of dominant model. Disease allele frequency of CNVs in the general population was obtained from Database for Genomic Variants (DGV) (http://dgv.tcag.ca/dgv/app/ home). The genotype relative risk was calculated from the OR obtained using the Fisher's exact test.

\section{Results \\ CNV-miRNA genes are enriched in SCZ patients with enhanced glycation/oxidative stress}

In this study, we compared the number of CNV-miRNAs between SCZ patients with high plasma pentosidine levels (PEN-SCZ) and those without the accumulation of plasma pentosidine (non-PEN-SCZ). Based on the power analysis, the expected power to detect significant CNV-miRNAs with an OR of 5.0 was 0.638 (Supplementary Table S1).

Ninety-four patients $(50.8 \%)$ with SCZ exhibited high plasma pentosidine levels, while 91 patients (49.2\%) did not; these were classified as the PEN-SCZ and non-PEN$\mathrm{SCZ}$ groups, respectively. We found a larger size of rare CNVs in the PEN-SCZ group, with 3.6 versus $0.4 \mathrm{Mb}$ for PEN-SCZ and non-PEN-SCZ, respectively (Table 1 and Supplementary Fig. S1). Additionally, the number of genes within CNVs was 20.5-fold higher in PEN-SCZ versus non-PEN-SCZ (Table 1 and Supplementary Fig. S2). Regarding the number of miRNAs within CNVs, it was also 9.8-fold higher in PEN-SCZ versus non-PEN-SCZ, suggesting that $\mathrm{CNV}$-miRNA genes were enriched in the context of PEN-SCZ (Table 1). On the other hand, although the number of miRNA genes within CNVs was larger in the PEN-SCZ group, the rate of patients harboring CNV-miRNAs was almost the same $(N=13)$. Next, we assessed the rate of miRNA genes in total genes within CNVs to adjust the effect of the CNV size; interestingly, miRNA-enriched CNVs, defined as per a miRNA-CNV rate $>50 \%$, were significantly higher in PEN-SCZ versus non-PEN-SCZ (Table 1 and Fig. $1, p=$ 0.032). Regarding the treatment resistant phenotype, clinical data from $98 \mathrm{SCZ}$ subjects were available. Among them, 22 patients $(39.3 \%)$ with PEN-SCZ were treatment resistant, and five patients (11.9\%) with non-PEN-SCZ presented treatment resistant phenotype $(p=0.0022)$. A summary of the individual miRNAs within CNVs in PENSCZ and non-PEN-SCZ is presented in Supplementary Table S2. Of note, one miRNA, miR-4768, overlapped between the PEN-SCZ and non-PEN-SCZ groups, suggesting that it has little contribution to the difference in the plasma pentosidine levels. Therefore, this miRNA was omitted from further analyses.

$\mathrm{GO}$ analysis suggests that the origin of the disruption in neurodevelopmental events is different in PEN-SCZ versus non-PEN-SCZ

A comparison of the $\mathrm{GO}$ properties of miRNAs within CNVs between PEN-SCZ and non-PEN-SCZ patients suggested that miRNAs involved in "midbrain dopamine neuron differentiation" $\left(14.3 \% ; 2.4 \times 10^{-7}\right)$ and "neuron-neuron synaptic transmission" (20.0\%; $1.2 \times$ $10^{-7}$ ) were likely to be affected in PEN-SCZ (Supplementary Table S3). On the other hand, "olfactory bulb interneuron differentiation," was affected in non-PENSCZ $\left(9.1 \% ; 3.2 \times 10^{-4}\right)$, suggesting that different molecular mechanisms may be behind the etiology of the two SCZ subtypes.

\section{Notable miRNAs in patients with PEN-SCZ}

Although the sample size and the number of observations were small, several notable CNV-miRNAs were identified in patients with PEN-SCZ with pentosidine plasma levels higher than $100 \mathrm{ng} / \mathrm{mL}$ in this study (Table 2). The main notable CNV-miRNAs are presented below.

\section{CNV-miRNAs involved in the regulation of CACNA1C and DRD2}

We identified one patient with PEN-SCZ presenting with extremely high plasma pentosidine levels ( $\mathrm{PEN}=505.4 \mathrm{ng} /$ ml, Table 2 and Supplementary Fig. S3). This patient developed SCZ at the age of 19 and was considered a treatment-resistant patient. The patient carried a single $\mathrm{CNV}$ at 11q14.11 (OR $=2.94,95 \% \mathrm{CI}: 0.12-73.01)$, 


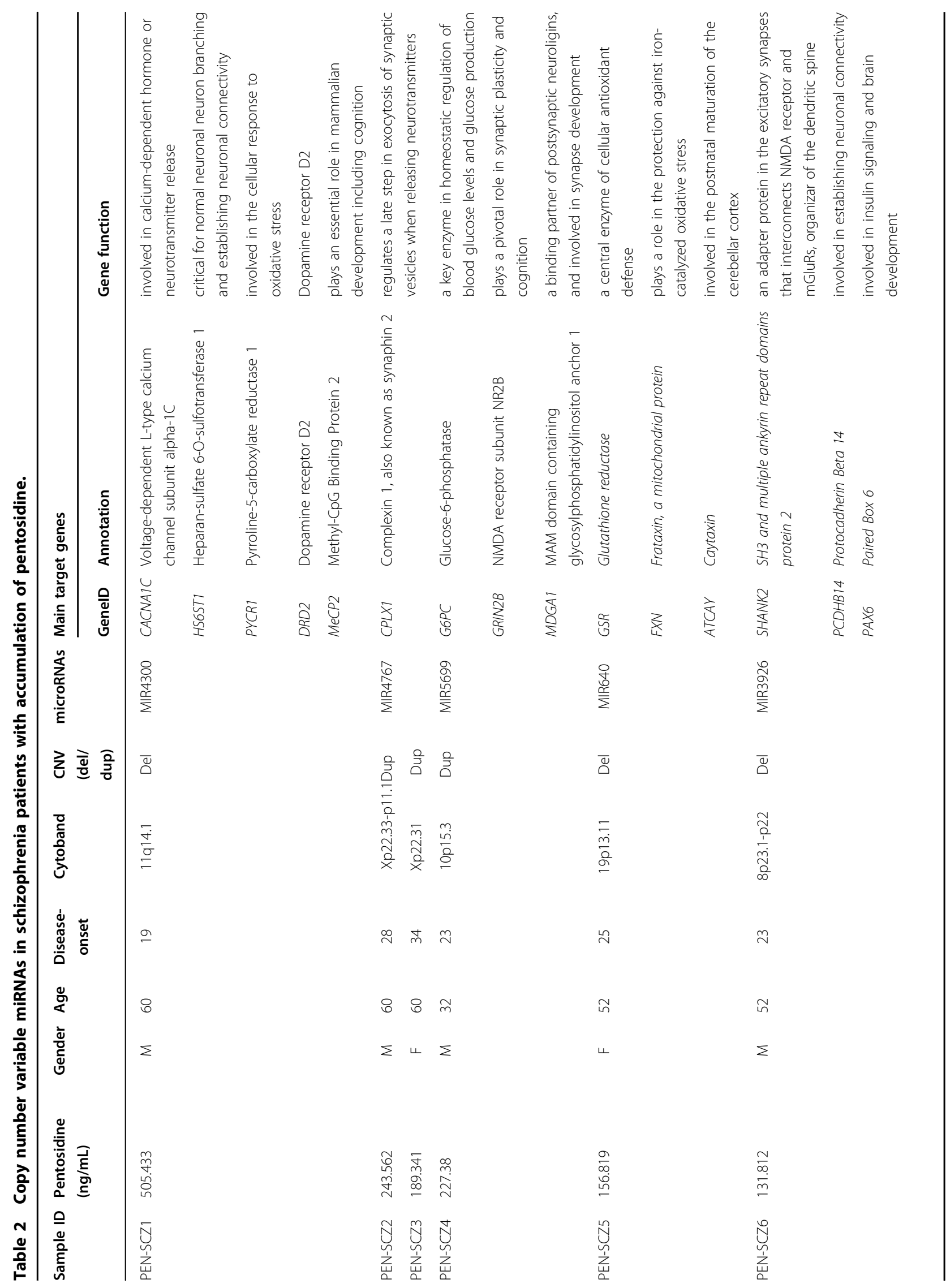


affecting only the miRNA-4300 (miR-4300) encoding gene, MIR4300. Interestingly, the in silico analysis identified 84 validated target genes of miR-4300 including CACNA1C, HS6ST1, and PYCR1 (Table 2). CACNA1C encodes voltage-dependent L-type calcium channel subunit alpha$1 \mathrm{C}$, which is involved in calcium-dependent hormone and neurotransmitter release. HS6ST1 encodes heparan-sulfate 6-O-sulfotransferase 1 , with roles in neuron branching and neuronal connectivity, critical for normal neuronal development. PYCR1 encodes pyrroline-5-carboxylate reductase 1 , which catalyzes the last step in the biosynthesis of proline and is involved in the cellular response to oxidative stress. The putative target genes of miR-4300 are listed in Supplementary Table S4, including DRD2 and MECP2 that encode dopamine receptor D2 and methyl-CpG binding protein 2 , respectively.

Notably, gene set enrichment analysis in the context of the validated target genes of miR-4300 suggested the enrichment of the following GO processes: (1) synaptic membrane adhesion to the extracellular matrix (27.3\%; $\left.5.5 \times 10^{-24}\right),(2)$ response to hormone $\left(40.0 \% ; 2.0 \times 10^{-8}\right)$, and (3) postsynaptic endosome to lysosome and postsynaptic neurotransmitter receptor diffusion $(26.0 \%$; $4.6 \times$ $10^{-34}$ ) (Supplementary Fig. S4). Additionally, the putative target genes of miR-4300 were also linked to "serotonin receptor signaling", one of the main targets of typical and atypical antipsychotic drugs.

\section{CNV-miRNA involved in glutamate signaling}

Sixty-nine CNV-miRNAs were found in at least two patients with PEN-SCZ. In particular, CNVs in the miR4767 gene, MIR4767, were shared in two patients with relatively high plasma pentosidine levels (PEN $>100 \mathrm{ng} /$ $\mathrm{ml}$, Table 2). Of note, given the small number of observations of this CNV-miRNA due to the limited sample size, the OR was 4.95 (95\%CI:0.23-104.45). Additionally, the in silico analysis revealed CPLX1 (encoding complexin 1 , also called synapsin 2 which regulates a late step in the exocytosis of synaptic vesicles) as a target gene of miR4767 (Table 2). Interestingly, gene set enrichment analysis of the target genes of miRNA-4767 suggested that miRNA-4767 may be involved in the "positive regulation of glutamate secretion" $\left(20.0 \%\right.$; $\left.9.5 \times 10^{-11}\right)$ (Table 3$)$.

\section{CNV-miRNAs are involved in glycation/oxidative stress and synaptic dysfunction}

Additionally, CNVs in genes encoding miR-5699, miR640, miR-3926, and miR-3156-5p may provide suggestive insights into the link between glycation/oxidative stress and synaptic dysfunction in the PEN-SCZ group (PEN $>100 \mathrm{ng} / \mathrm{mL}$ ) (Table 2). The notable targets of these miRNAs are presented in Table 2. MiR-5699 was disrupted by CNVs in a PEN-SCZ patient who exhibited $277.38 \mathrm{ng} / \mathrm{ml}$ plasma pentosidine (Table 2). The validated 
Table 3 Gene set enrichment analysis of target genes for the copy number variable miRNAs in schizophrenia patients with accumulation of pentosidine.

\begin{tabular}{|c|c|c|}
\hline \multirow[t]{2}{*}{ Disrupted miRNAs } & \multicolumn{2}{|l|}{ Pathways affected by target genes disrupted by CNV-miRNAs } \\
\hline & Validated & Putative \\
\hline \multirow[t]{3}{*}{ miR-4300 } & $\begin{array}{l}\text { Synaptic membrane adhesion to extracellular matrix (27.3\%; } 5.5 \times \\
\left.10^{-24}\right)\end{array}$ & $\begin{array}{l}\text { G protein-coupled serotonin receptor signaling } \\
\text { pathway }\left(22.7 \% ; 4.1 \times 10^{-19}\right)\end{array}$ \\
\hline & Response to hormone $\left(40.0 \% ; 2.0 \times 10^{-8}\right)$ & Serotonin receptor signaling $\left(22.7 \% ; 1.7 \times 10^{-18}\right)$ \\
\hline & $\begin{array}{l}\text { Postsynaptic endosome to lysosome, postsynaptic neurotransmitter } \\
\text { receptor diffusion }\left(26.0 \% ; 4.6 \times 10^{-34}\right)\end{array}$ & Cognition $\left(40.9 \% ; 3.1 \times 10^{-18}\right)$ \\
\hline \multirow[t]{6}{*}{ miR-4767 } & Negative regulation of establishment of endothelial barrier & Positive regulation of neuron death \\
\hline & $\left(13.0 \% ; 8.7 \times 10^{-10}\right)$ & $\left(36.7 \% ; 5.6 \times 10^{-25}\right)$ \\
\hline & Neuropeptide signaling pathway & Positive regulation of neuron apoptotic process \\
\hline & $\left(21.7 \% ; 2.6 \times 10^{-7}\right)$ & $\left(32.7 \% ; 8.3 \times 10^{-25}\right)$ \\
\hline & Positive regulation of glutamate secretion & \\
\hline & $\left(20.0 \% ; 9.5 \times 10^{-11}\right)$ & \\
\hline \multirow[t]{6}{*}{ miR-5699 } & Synaptic membrane adhesion to extracellular matrix & Neuropeptide signaling pathway \\
\hline & $\left(21.7 \% ; 1.0 \times 10^{-25}\right)$ & $\left(32.0 \% ; 1.6 \times 10^{-12}\right)$ \\
\hline & Synaptic vesicle cytoskeletal transport & Circadian sleep/wake cycle process \\
\hline & $\left(17.4 \% ; 2.7 \times 10^{-17}\right)$ & $\left(20.0 \% ; 7.9 \times 10^{-13}\right)$ \\
\hline & & G protein-coupled receptor signaling pathway \\
\hline & & $\left(60.0 \% ; 3.9 \times 10^{-10}\right)$ \\
\hline \multirow[t]{4}{*}{ miR-640 } & Negative regulation of insulin secretion response to glucose stimulus & GABAergic synaptic transmission \\
\hline & $\left(25.0 \% ; 1.2 \times 10^{-12}\right)$ & $\left(18.0 \% ; 2.0 \times 10^{-14}\right)$ \\
\hline & Long-term synaptic potentiation & Response to oxidative stress \\
\hline & $\left(25.0 \% ; 6.2 \times 10^{-9}\right)$ & $\left(33.3 \% ; 1.2 \times 10^{-9}\right)$ \\
\hline \multirow[t]{5}{*}{ miR-3926 } & $N / A^{*}$ & Glutamate receptor signaling pathway \\
\hline & & $\left(42.9 \% ; 1.9 \times 10^{-12}\right)$ \\
\hline & & $\begin{array}{l}\text { Wnt signaling pathway involved in midbrain } \\
\text { dopaminergic neuron differentiation }\end{array}$ \\
\hline & & $\left(17.4 \% ; 7.5 \times 10^{-10}\right)$ \\
\hline & & Glucose metabolic process $\left(23.5 \% ; 6.5 \times 10^{-6}\right)$ \\
\hline \multirow[t]{4}{*}{ miR-3156-5p } & $\begin{array}{l}\text { Cellular response to phenylpropanoid (antioxidants) (100.0\%; } 2.6 \times \\
\left.10^{-4}\right)\end{array}$ & Positive regulation of neuron death $\left(44.0 \% ; 8.8 \times 10^{-17}\right)$ \\
\hline & $\begin{array}{l}\text { Cellular response to hydroxyisoflavone (antioxidants) (100.0\%; } 2.6 \times \\
10^{-4} \text { ) }\end{array}$ & GABA signaling pathway $\left(56.0 \% ; 1.1 \times 10^{-16}\right)$ \\
\hline & Cellular response to genistein (antioxidants) $\left(100.0 \% ; 2.6 \times 10^{-4}\right)$ & \\
\hline & Gamma-aminobutyric acid secretion $\left(12.5 \% ; 4.7 \times 10^{-13}\right)$ & \\
\hline
\end{tabular}

*Validated gene targets were not available.

target genes of miR-5699 included G6PC, GRIN2B, and $M D G A 1$ (Table 2). G6PC encodes glucose-6-phosphatase, a key enzyme responsible for glucose production; GRIN2B encodes the NMDA receptor subunit NR2B that plays a pivotal role in synaptic plasticity and cognition; MDGA1 encodes a MAM domain containing the glycosylphosphatidylinositol anchor 1, known as a negative regulator of synapse development and its expression is brainspecific (Supplementary Fig. S5). Of note, gene set enrichment analysis of the miR-5699 target genes 
implicated the possible involvement of the "synaptic membrane adhesion to the extracellular matrix," "sleep," and "regulation of neuronal death" processes (Table 3).

CNVs in the miR-640 gene (MIR640) were also found in one patient with PEN-SCZ (OR $=2.94,95 \% \mathrm{CI}$ : 0.12-73.01). Importantly, oxidative stress genes, including GSR and FXN, were included as its validated targets (Table 2). GSR encodes glutathione reductase, a central enzyme of the cellular antioxidant defense. FXN encodes frataxin, a mitochondrial protein that plays a role in the protection of neurons against iron-catalyzed oxidative stress at puberty. Another validated target of miR-640 was $A T C A Y$, which encodes caytaxin, involved in the postnatal maturation of the cerebellar cortex. Of note, based on the gene set enrichment analysis, the validated miR-640 target genes were enriched in the pathways involved in the "negative regulation of insulin secretion in response to glucose stimulus" $\left(25.0 \% ; 1.2 \times 10^{-12}\right)$ and "long-term synaptic potentiation $\left(25.0 \% ; 6.2 \times 10^{-9}\right)$," suggesting the possible link between glycation stress and synaptic function (Table 3). Additionally, the putative miR-640 target genes were enriched in "GABAergic synaptic transmission" $\left(18.0 \% ; 2.0 \times 10^{-14}\right)$ and "response to oxidative stress" (33.3\%; $1.2 \times 10^{-9}$, Table 3$)$.

MiR-3926 was also noted in one patient with PEN-SCZ (Table 2). Although no validated target genes were identified as per in silico analysis, predicted target genes included SHANK2, PCDHB14, and PAX6. SHANK2 encodes $\mathrm{SH} 3$ and multiple ankyrin repeat domains protein 2 , an adapter protein in the postsynaptic density of excitatory synapses that interconnects postsynaptic NMDA-type and metabotropic glutamate receptors (mGluRs); SHANK2 plays a pivotal role in the organization of the dendritic spine; $P C D H B 14$, encoding protocadherin beta 14, plays a critical role in the establishment of neuronal connectivity; PAX6 encodes paired box 6 and is involved in insulin signaling and brain development (Table 2). The expression of PAX6 is brain-specific (Supplementary Fig. S6). In line with these results, according to the gene set enrichment analysis, pathways related to synaptic function were enriched, such as the "glutamate receptor signaling pathway" $(42.9 \% ; 1.9 \times$ $10^{-12}$, Table 3).

MiR-3156-5p, possibly involved in GABA signaling and oxidative stress was also observed in one patient with PEN-SCZ. The validated target genes of miR-3156-5p included TXNL1, encoding thioredoxin like 1, a member of the antioxidative thioredoxin system (Table 2). Gene set enrichment analysis on miR-3156-5p target genes suggested their involvement in GABA receptor signaling and oxidative stress, similar to the results obtained in the context of miR-640 (Table 3). The comprehensive list of the validated and putative miRNA target genes is provided in Supplementary Table S5.

\section{Discussion}

In the present study, we examined the frequency of CNV-miRNAs in SCZ with and without accumulated plasma pentosidine, including the GO properties regulated by those miRNAs. Gene set enrichment analysis in the context of miRNA target genes found in rare CNVs was further performed for a better understanding of the molecular pathogenesis of PEN-SCZ. As a result, we observed that PEN-SCZ harbored 9.8-fold more miRNAenriched CNVs than non-PEN-SCZ, possibly affecting different neuronal developmental events. In fact, analyses of individual CNV-miRNAs in PEN-SCZ suggested that miRNAs related to glycation/oxidative stress, brain maturation, and synaptic network, especially glutamate/ GABA receptor signaling may be affected. Of note, although our sample size was limited, several notable CNV-miRNAs of interest were observed and suggestive evidence potentially linking glycation/oxidative stress and synaptic function via CNV-miRNAs was modestly disclosed.

Previously, it has been reported that more miRNAs are disrupted by rare CNVs in SCZ patients compared to $\operatorname{controls}^{19}$. For example, Warnica et al reported that patients with SCZ harbored 3.29-fold more CNVmiRNAs $^{19}$. In this study, we found that 9.8-fold more miRNA genes were affected by rare CNVs in patients with PEN-SCZ versus non-PEN-SCZ, suggesting that the dysregulation of miRNAs may also be involved in the etiology of PEN-SCZ. Given that PEN-SCZ patients were reported to exhibit a clinical phenotype resistant to the current antipsychotic treatments ${ }^{23,24}$ and so as in our cohort (Table 1), we hypothesize this might be due to the high number of CNV-miRNA genes affecting multiple pathways. One fact potentially supporting this hypothesis is the deletion of MIR4300: the expression of DRD2, targeted by miR-4300, was possibly upregulated, making this patient resistant to the current antipsychotic drugs which mainly block DRD2.

Regarding the GO analysis, a previous CNV-miRNA study reported that neurodevelopmental pathways including axonogenesis and neuron projection development were affected in $\mathrm{SCZ}^{19}$. Our study, particularly the GO analysis, supports this notion that neuronal developmental events may be affected via CNV-miRNAs in SCZ. Interestingly, we found that the origins of the phenotype appeared to be different between PEN-SCZ and non-PEN-SCZ, which may also be behind the resistance to treatment with antipsychotic drugs in PEN-SCZ.

Regarding the notable CNV-miRNAs identified in this study, MIR4300 has been reported as a risk gene for adolescent idiopathic scoliosis (AIS) ${ }^{33}$ as well as for SCZ in the latest large-scale GWAS ${ }^{7}$. Here, we identified the deletion of the entire MIR4300 in a patient with SCZ showing extremely high plasma pentosidine. It is 
interesting to note that the onset during adolescence is a shared feature between AIS and SCZ. MIR4300 is highly expressed in the testis and brain and thought to bind to the hormone regulating genes associated with the diseaseonset during adolescence ${ }^{33}$. Our gene set enrichment analysis results for the miR-4300 target genes supported this notion, showing its involvement in hormonal regulation. In fact, of the 14 novel genome-wide significant loci identified in the large-scale GWAS on AIS, using 79,211 Japanese individuals, four genes overlapped with SCZ risk genes, namely CSMD1, PLXNA2, MTMR11, and $C D K 13^{7,8,34}$. In addition, the target genes of miR-4300 included CACNA1C, DRD2, MECP2, and PYCR1, the most recurrent SCZ risk genes ${ }^{7,8,35}$. Under normal conditions, miR-4300 binds to the $3^{\prime} \mathrm{UTR}$ of CACNA1C and negatively regulates its expression, resulting in protection against oxidative stress ${ }^{36}$. Although in this study we identified only one case, we still speculate that SCZ patients harboring the entire deletion of MIR4300 lack this protective mechanism and are vulnerable to oxidative stress. PYCR1, another miR-4300 target gene that is linked to glycation stress ${ }^{37}$, interacts with DJ-1, one of the deglycation critical enzymes, and prevents neuronal damage caused by the accumulation of advanced glycation end product (AGE) like pentosidine, the marker of glycation $^{37,38}$. Therefore, the deletion of MIR4300 might be involved in both enhanced glycation stress and oxidative stress through PYCR1 and CACNA1C (Fig. 2).

Moreover, we identified G6PC, a key glucose metabolism enzyme, and GSR, the main enzyme of the antioxidative stress system, as the validated miRNA target genes in SCZ patients with accumulated plasma pentosidine. Pentosidine is continuously formed; however, this occurs more rapidly under excess oxidative stress and hyperglycemia, making it a biomarker of both glycation and oxidative damage to proteins ${ }^{39}$ In this context, it is particularly interesting that enzymes involved in glucose metabolism and oxidative stress were both targeted by the CNV-miRNAs in SCZ patients with high plasma pentosidine.

In accordance with the NMDA receptor hypofunction hypothesis ${ }^{40,41}$, GRIN2B, encoding the NMDA receptor subunit NR2B, was identified as a validated target gene of miR-5699, affected in patients with PEN-SCZ. Additionally, in this study, another patient with high plasma pentosidine harbored the deletion of the entire MIR-3926 that targets $S H A N K 2$, an adapter protein that interconnects postsynaptic NMDA receptor and mGluR $5^{42}$. We might, therefore, speculate that the shared miRNA target genes are related to NMDA receptor signaling molecules, while the other target genes may contribute to generate the heterogeneous phenotypes observed in SCZ.

Interestingly, in the context of miR-640 and miR-3156$5 p$, similar pathways were enriched as per the miRNA target genes, such as GABAergic synaptic neurotransmission and oxidative stress (Table 3). Methylglyoxal, a key precursor of AGEs such as pentosidine ${ }^{43}$, acts as a GABAA receptor partial agonist ${ }^{44}$. Furthermore, previous studies reported that the GABAergic neurons are more vulnerable to oxidative stress ${ }^{45}$, raising the possibility that they might be also vulnerable to glycation stress. The high energy demand for the presynaptic release of GABA was previously discussed in brain diseases ${ }^{46}$. Our results may suggest that miRNAs targeting the GABAA receptor signaling pathway are disrupted by CNVs in a portion of SCZ patients with high plasma levels of pentosidine. Figure 2 illustrates a possible link between glycation/oxidative stress and the synaptic function mediated by CNV-miRNAs.

This study has several limitations. First, the sample size was small and the study was underpowered (Supplementary Table S1) as we analyzed CNV-miRNAs using patients with SCZ harboring rare CNVs. Although the clinical evaluation of the relationship between genomic factors and phenotypes in individual patients is needed to generate more data, a larger sample size is required in order not to miss other important CNV-miRNAs. Of note, evaluating the effect size of CNVs that are too rare to study in individual association studies has been in deep argument ${ }^{47}$. Of note, GO and gene set enrichment analyses may help to understand the pathway affected by the rare CNV-miRNAs, the reason why we performed them in this study. Second, our analyses included not only validated target genes for the miRNAs but also putative genes. The need for the prediction of miRNA target genes using an in silico computational approach is another limitation of the present study. Third, the cause of the increase in plasma pentosidine may be polygenic in nature and attributed to the sum of small individual effects. Although miR-4300 was identified as only one gene affected by CNVs, other genes in CNVs not coding for miRNAs coexisted suggesting that the etiology of SCZ is associated with a combined effect of CNVs on miRNAs and other genes. Forth, the possibility that other genetic and epigenetic factors such as methylation was not excluded.

In conclusion, although this is a preliminary study with a limited sample size, our integrative genome-wide CNVmiRNA analysis is the first to provide suggestive evidence that miRNA-enriched CNVs are more prevalent in PEN$\mathrm{SCZ}$ versus non-PEN-SCZ, disrupting different neuronal developmental events. Of note, the analysis of individual CNV-miRNAs observed in PEN-SCZ implicated a possible regulatory link between glycation/oxidative stress and synaptic dysfunction in SCZ patients with enhanced glycation/oxidative stress. Therefore, targeting miRNAs within CNVs to restore the aberrant synaptic function may be a promising strategy for the development of 


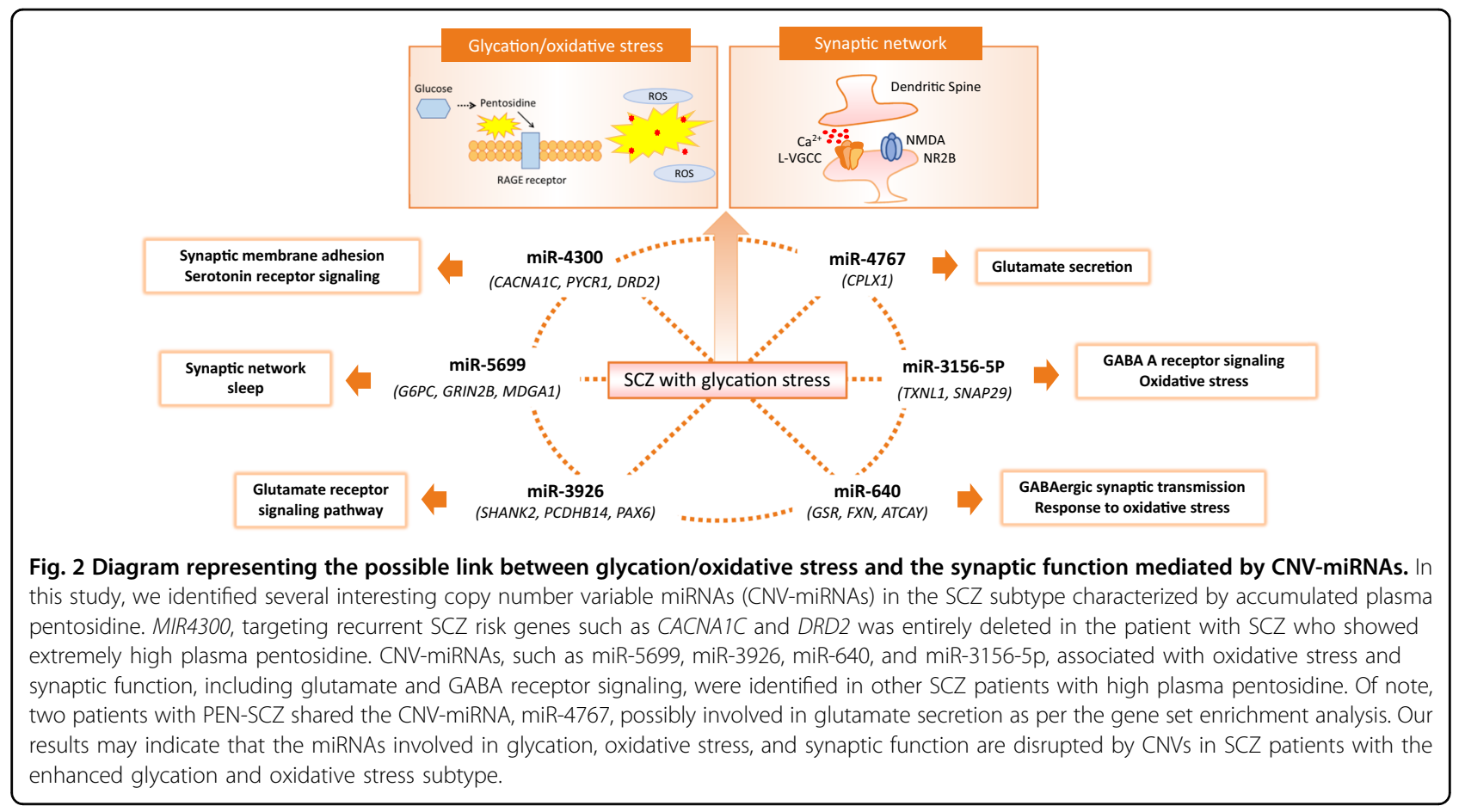

personalized treatments for SCZ patients with enhanced glycation/oxidative stress.

\section{Acknowledgements}

We are grateful for the expert technical assistance of Nanako Obata, Izumi Nohara, Mai Hatakenaka, Yukiko Shimada, Chikako Ishida, and Ikuyo Kito. We especially thank Hiroko Yuzawa at Tokai University School of Medicine for the measurement of pentosidine. We are grateful to all of the families who took part in this study. This work was supported by JSPS KAKENHI grant numbers $17 \mathrm{H} 05090$ and 15K19720 (Dr. Kushima), 18K07579 (Dr. Miyashita), 16H05380 17H05930, 19H04887, and 20H03608 (Dr. Arai), and 19K17075 (Dr. Yoshikawa); the Japan Agency for Medical Research and Development (AMED) under grant numbers JP20km0405216, JP20ek0109411, JP21 km0405216, JP21ek0109411, JP20dm0107087, JP20dm0207075, and JP20dm0107160 (Dr. Kushima), JP20ak0101113, JP20ak0101126, JP20dk0307075, JP20dm0207075, and JP20dm0107087 (Dr. Ozaki), JP20dm0107088 (Dr. Itokawa); the Uehara Memorial Foundation (Dr. Arai); and the Sumitomo Foundation (Dr. Arai).

\section{Author details}

${ }^{1}$ Schizophrenia Research Project, Department of Psychiatry and Behavioral Sciences, Tokyo Metropolitan Institute of Medical Science, 2-1-6, Kamikitazawa, Setagaya-ku 156-8506 Tokyo, Japan. ²Department of Adolescent Psychiatry, Nihonbashi Sun Clinic, 2-1-21, Nihonbashi, Chuou-ku, Tokyo 103-0027, Japan. ${ }^{3}$ Department of Psychiatry, Nagoya University Graduate School of Medicine, 65 Tsurumai, Showa-ku, 466-8550 Nagoya, Aichi, Japan. ${ }^{4}$ Medical Genomics Center, Nagoya University Hospital, Nagoya, Aichi 466-8550, Japan. ${ }^{5}$ Department of Psychiatry, Takatsuki Clinic, Tanaka-cho, 196-0014 Akishima, Tokyo, Japan. ${ }^{6}$ Department of Psychiatry, Tokyo Metropolitan Matsuzawa Hospital, 2-1-1 Kamikitazawa, Setagaya-ku 156-0057 Tokyo, Japan. ${ }^{7}$ Research Center for Genome \& Medical Sciences, Tokyo Metropolitan Institute of Medical Science, 2-1-6, Kamikitazawa, 156-8506 Setagaya-ku, Tokyo, Japan. ${ }^{8}$ Division of Neurology, Tokai University School of Medicine, 143 Shimo-Kasuya, 259-1193 Isehara, Kanagawa, Japan

\section{Author contributions}

A.Y. and M.A. designed the study; M.A. and M.I. supervised the writing of the manuscript. A.Y., I.K., and M.A. performed the experimental work and undertook the statistical analysis. A.Y. wrote the first draft of the manuscript. All authors participated in the collection of blood samples from patients and helped complete this manuscript. All authors have approved the final version of the manuscript.

\section{Conflict of interest}

The authors declare no competing interests.

\section{Publisher's note}

Springer Nature remains neutral with regard to jurisdictional claims in published maps and institutional affiliations.

Supplementary information The online version contains supplementary material available at https://doi.org/10.1038/s41398-021-01460-1.

Received: 19 August 2020 Revised: 30 April 2021 Accepted: 17 May 2021 Published online: 28 May 2021

\section{References}

1. Friston, K. Disconnection and cognitive dysmetria in schizophrenia. A. J. Psychiatry 162, 429-432 (2005).

2. Petronis, A. The origin of schizophrenia: genetic thesis, epigenetic antithesis, and resolving synthesis. Biol. Psychiatry 55, 965-970 (2004).

3. Consortium, I. S. Rare chromosomal deletions and duplications increase risk of schizophrenia. Nature 455, 237 (2008).

4. Consortium, I. S. Common polygenic variation contributes to risk of schizophrenia that overlaps with bipolar disorder. Nature 460, 748 (2009).

5. Stefansson, $\mathrm{H}$. et al. Common variants conferring risk of schizophrenia. Nature 460, 744-747 (2009).

6. Montano, C. et al. Association of DNA methylation differences with schizophrenia in an epigenome-wide association study. JAMA Psychiatry 73, 506-514 (2016).

7. Ikeda, M. et al. Genome-wide association study detected novel susceptibility genes for schizophrenia and shared trans-populations/diseases genetic effect. Schizophr. Bull. 45, 824-834 (2019).

8. Ripke, S. et al. Biological insights from 108 schizophrenia-associated genetic loci. Nature 511, 421-427 (2014). 
9. Genovese, G. et al. Increased burden of ultra-rare protein-altering variants among 4,877 individuals with schizophrenia. Nat. Neurosci. 19, 1433-1441 (2016).

10. Singh, T. et al. The contribution of rare variants to risk of schizophrenia in individuals with and without intellectual disability. Nat. Genet. 49, 1167-1173 (2017).

11. Stefansson, $\mathbf{H}$. et al. Large recurrent microdeletions associated with schizophrenia. Nature 455, 232-236 (2008).

12. Malhotra, D. et al. High frequencies of de novo CNVs in bipolar disorder and schizophrenia. Neuron 72, 951-963 (2011)

13. Bassett, A. S. et al. Rare genome-wide copy number variation and expression of schizophrenia in 22q11. 2 deletion syndrome. A. J. Psychiatry 174, 1054-1063 (2017)

14. Marshall, C. R. et al. Contribution of copy number variants to schizophrenia from a genome-wide study of 41,321 subjects. Nat. Genet. 49, 27-35 (2017).

15. Nakatochi, M., Kushima, I. \& Ozaki, N. Implications of germline copy-number variations in psychiatric disorders: review of large-scale genetic studies. J. Hum. Genet. 66, 1-13 (2020).

16. Kushima, I. et al. Comparative analyses of copy-number variation in autism spectrum disorder and schizophrenia reveal etiological overlap and biological insights. Cell Rep. 24, 2838-2856 (2018).

17. Bartel, D. P. MicroRNAs: genomics, biogenesis, mechanism, and function. Cell 116, 281-297 (2004).

18. Marcinkowska, M., Szymanski, M., Krzyzosiak, W. J. \& Kozlowski, P. Copy number variation of microRNA genes in the human genome. BMC Genomics 12, 183 (2011).

19. Warnica, W. et al. Copy number variable microRNAs in schizophrenia and their neurodevelopmental gene targets. Biol. Psychiatry 77, 158-166 (2015).

20. Vaishnavi, V., Manikandan, M., Tiwary, B. K. \& Munirajan, A. K. Insights on the functional impact of microRNAs present in autism-associated copy number variants. PloS ONE 8, e56781 (2013).

21. Carpenter, W. T. Jr \& Kirkpatrick, B. The heterogeneity of the long-term course of schizophrenia. Schizophr. Bull. 14, 645-652 (1988).

22. Eichler, E. E. et al. Missing heritability and strategies for finding the underlying causes of complex disease. Nat. Rev. Genet. 11, 446-450 (2010).

23. Arai, M. et al. Enhanced carbonyl stress in a subpopulation of schizophrenia. Arch. Gen. Psychiatry 67, 589-597 (2010).

24. Miyashita, M. et al. Clinical features of schizophrenia with enhanced carbonyl stress. Schizophr. Bull. 40, 1040-1046 (2014).

25. Miyata, T. et al. Identification of pentosidine as a native structure for advanced glycation end products in beta-2-microglobulin-containing amyloid fibrils in patients with dialysis-related amyloidosis. Proc. Natl Acad. Sci. USA 93, 2353-2358 (1996).

26. Sekiguchi, M. et al. ARHGAP10, which encodes Rho GTPase-activating protein 10, is a novel gene for schizophrenia risk. Transl. Psychiatry 10, 1-15 (2020).

27. Kushima, I. et al. High-resolution copy number variation analysis of schizophrenia in Japan. Mol. Psychiatry 22, 430-440 (2017).

28. Chen, Y. \& Wang, X. miRDB: an online database for prediction of functional microRNA targets. Nucleic Acids Res. 48, D127-D131 (2020).

29. Sticht, C., De La Torre, C., Parveen, A. \& Gretz, N. miRWalk: an online resource for prediction of microRNA binding sites. PloS ONE 13, e0206239 (2018).
30. Szklarczyk, D. et al. STRING v11: protein-protein association networks with increased coverage, supporting functional discovery in genome-wide experimental datasets. Nucleic Acids Res. 47, D607-D613 (2019).

31. Sivachenko, A. Y. \& Yuryev, A. Pathway analysis software as a tool for drug target selection, prioritization and validation of drug mechanism. Expert Opin. Ther. Targets 11, 411-421 (2007).

32. Skol, A. D., Scott, L. J., Abecasis, G. R. \& Boehnke, M. Joint analysis is more efficient than replication-based analysis for two-stage genome-wide association studies. Nat. Genet. 38, 209-213 (2006).

33. Ogura, Y. et al. A functional variant in MIR4300HG, the host gene of microRNA MIR4300 is associated with progression of adolescent idiopathic scoliosis. Hum. Mol. Genet. 26, 4086-4092 (2017).

34. Kou, I. et al. Genome-wide association study identifies 14 previously unreported susceptibility loci for adolescent idiopathic scoliosis in Japanese. Nat. Commun. 10, 1-9 (2019).

35. Kwon, E., Wang, W. \& Tsai, L. Validation of schizophrenia-associated genes CSMD1, C10orf26, CACNA1C and TCF4 as miR-137 targets. Mol. Psychiatry 18, $11-12(2013)$

36. Michels, S., W"hr, M., Schwarting, R. K. \& Culmsee, C. Psychiatric risk gene Cacna1c determines mitochondrial resilience against oxidative stress in neurons. Cell Death Dis 9, 645 (2018).

37. Yasuda, T. et al. DJ-1 cooperates with PYCR1 in cell protection against oxidative stress. Biochem. Biophys. Res. Commun. 436, 289-294 (2013).

38. Richarme, G. et al. Parkinsonism-associated protein DJ-1/Park7 is a major protein deglycase that repairs methylglyoxal-and glyoxal-glycated cysteine, arginine, and lysine residues. J. Biol. Chem. 290, 1885-1897 (2015).

39. Thornalley, P. J. The glyoxalase system in health and disease. Mol. Asp. Med. 14, 287-371 (1993).

40. Moghaddam, B. \& Javitt, D. From revolution to evolution: the glutamate hypothesis of schizophrenia and its implication for treatment. Neuropsychopharmacology 37, 4-15 (2012)

41. Snyder, M. A. \& Gao, W.-J. NMDA receptor hypofunction for schizophrenia revisited: perspectives from epigenetic mechanisms. Schizophr. Res. 217, 60-70 (2019).

42. Naisbitt, S. et al. Shank, a novel family of postsynaptic density proteins that binds to the NMDA receptor/PSD-95/GKAP complex and cortactin. Neuron 23 569-582 (1999).

43. Chaudhuri, J. et al. The role of advanced glycation end products in aging and metabolic diseases: bridging association and causality. Cell Metab. 28, 337-352 (2018).

44. Distler, M. G. et al. Glyoxalase 1 increases anxiety by reducing GABA A receptor agonist methylglyoxal. J. Clin. Investig. 122, 2306-2315 (2012).

45. Steullet, P. et al. Oxidative stress-driven parvalbumin interneuron impairment as a common mechanism in models of schizophrenia. Mol. Psychiatry 22, 936-943 (2017).

46. Kann, $\mathrm{O}$. The interneuron energy hypothesis: implications for brain disease. Neurobiol. Dis. 90, 75-85 (2016).

47. Huguet, G. et al. Measuring and estimating the effect sizes of copy number variants on general intelligence in community-based samples. JAMA Psychiatry 75, 447-457 (2018). 